\title{
The Study of Regional R\&D Innovation Efficiency Based on Improved Two-Stage DEA Model: Evidence from 30 Provinces of China
}

\author{
Yuyu Li ${ }^{1, *}$, Bo Huang ${ }^{2, ~ * ~ B o ~ F u ~}{ }^{3}$ \\ ${ }^{1}$ College of Computer and Information Science, Chongqing Normal University, Chongqing, China \\ ${ }^{2}$ School of Economics and Business Administration, Chongqing University, Chongqing, China \\ ${ }^{3}$ Shanghai Pudong Development Bank Co Ltd Qingdao Branch, Qingdao, China
}

\section{Email address:}

lyyjame@163.com (Yuyu Li), 395532389@qq.com (Bo Huang), fubo_job@163.com (Bo Fu)

${ }^{*}$ Corresponding author

\section{To cite this article:}

Yuyu Li, Bo Huang, Bo Fu. The Study of Regional R\&D Innovation Efficiency Based on Improved Two-Stage DEA Model: Evidence from 30 Provinces of China. Science Innovation. Vol. 6, No. 2, 2018, pp. 80-86. doi: 10.11648/j.si.20180602.15

Received: April 21, 2018; Accepted: June 12, 2018; Published: June 22, 2018

\begin{abstract}
The improved two-stage DEA model removed constrains that efficiency values of decision making units are not greater than one by means of the handling method in super-BCC model. In this way, those efficient DMUs were separated from the efficient frontier and therefore the problem that they were unable to sort in the traditional two-stage DEA model. In the meantime, this improved model gave full consideration to the dual role of intermediate outputs and the influence of scale effect, which gave the evaluation results larger reference value. Lastly, the case study of 30 provinces demonstrated the feasibility and rationality of the improved model. It is found that the level of research and innovation efficiency in east China is the highest; the comprehensive efficiency and stage efficiency of mid-south and north China are high; the comprehensive efficiency level in southwest and northwest China is high while the efficiency of scientific research and development is low; the efficiency of research and innovation in northeast China is lowest.
\end{abstract}

Keywords: Improved Two-Stage DEA Model, Ranking of Efficient DMUs, Intermediate Outputs, Dual Role, Scale Effect

\section{基于改进两阶段DEA模型的区域研发创新效率研究——来自30省 份的实证数据}

\author{
李宇雨 ${ }^{1 *}$, 黄波 ${ }^{2 *}$, 傅博 ${ }^{3}$ \\ 1计算机与信息科学学院, 重庆师范大学, 重庆, 中国 \\ 2经济与工商管理学院, 重庆大学, 重庆, 中国 \\ ${ }^{3}$ 上海浦东发展银行股份有限公司青岛分行, 青岛, 中国
}

\section{邮箱}

lyyjame@163.com（李宇雨），395532389@qq.com（黄波），fubo_job@163.com（傅博） 
地区研发创新效率水平最高, 中南、华北的综合效率和分阶段效率均高, 西南、西北地区综合效率水平高, 但科技研 发效率或经济转化效率低，东北地区研发创新效率最低。

关键词：改进两阶段DEA模型，有效DMU排序，中间产出，双重角色，规模效应

\section{1. 引言}

数据包络分析（Data Envelopment Analysis, DEA） 作为一种借助数学规划建模的非参数分析方法, 已经被广 泛地运用到效率评价的诸多领域中，推动了理论进步与实 践发展。随着传统单阶段DEA模型运用中“黑箱”问题导致 的效率评价不准确甚至失效等问题涌现，人们逐渐认识到 效率评价过程中对系统内部运作流程进行清晰化、深入化 分析的重要性, 因此两阶段DEA模型应运而生。

两阶段DEA模型的提出实现了对评价系统内部结构 和子过程的细化分析, 使得评价结果更有效。目前许多学 者已经对相关研究进行了一定的探索, Yao等将信息技术 投资收益的效率分为两个阶段, 利用“算数平均”的方法组 合两个子效率, 实现对总体效率的测度 [1]。Kao等提出对 关联两阶段的投入进行分配，在考虑初始投入对两阶段的 不同作用的基础上进行建模[2]。陈凯华等分别在固定规模 报酬与可变规模报酬假设下, 构建了存在共享投入的两阶 段DEA效率评价模型, 充分考虑了中间产品的双重作用[3]。 冯锋等在建模过程中考虑创新过程的阶段滞后性特点, 采 用链式网络DEA的方法对 29 个省市的科技投入产出数据 进行了研究, 比较了省区和区域研发效率的差异性[4]。马 建峰等运用包含共享投入和中间产出的两阶段DEA模型 实现了对中国大中型工业企业技术创新效率的测度 [5]。总 体来看, 两阶段DEA效率评价模型已经被广泛地应用于商 业银行[6]、保险公司[7]、工业企业[8]等的运营效率评价 以及区域研发效率评价等多个领域, 有关子过程与总体效 率关系的测度方法主要包括“算数平均”[1]、“几何平均”[9]、 “加法模型”[10]和“乘法模型”[11]等, 实现了对有效DMU 和无效DMU的分离。然而, 当前两阶段DEA效率评价模
型无法实现对有效性决策单元的排序, 因此无法对有效性 决策单元进行进一步分析比较, 给现实的评价过程带来了 一定的问题。

鉴于这一背景，本文借鉴Andersen等[12]提出的将有 效决策单元从效率前沿面分离出去的思路, 修改两阶段 DEA模型中对决策单元的约束条件, 解决当前效率评价过 程中无法对有效DMU进行排序的问题，此外，在模型构 建过程中考虑规模效应的影响与中间产出的“双重角色”, 以期实现对传统两阶段DEA效率评价模型的优化。最后, 为了对模型的可行性进行论证, 运用 30 个省份的数据对模 型进行实证分析, 利用所得分析结果对区域间研发创新效 率与水平进行比较, 以期对不同区域研发创新的策略制定 提供一点参考。

\section{2. 两阶段研发创新过程描述}

考虑如图1所示的两阶段研发创新过程, 分为科技研 发和经济转化两个阶段。假设有 $n$ 个决策单元 (decision making unit, DMU) , 每个DMU $(j=1,2 \ldots \ldots n)$ 有 $m$ 个初 始投入, 分为两类: $X_{i j}\left(i=1,2, \cdots \cdots, m_{0}\right)$ 和 $X_{l j}\left(l=m_{0}+1, \cdots \cdots, m\right)$ $\left(m_{0} \leq m\right)$ ，其中 $X_{i j}$ 为两阶段共享型投入，两阶段的分配 比例分别为 $\alpha_{i}$ 和 $\left(1-\alpha_{i}\right) \quad\left(0<\alpha_{i} \leq 1\right), X_{l j}$ 为第一阶段单独 投入。每个 $\mathrm{DMU}_{j}$ 有 $q$ 个中间产出 $Z_{p j}(p=1,2 \ldots \ldots q)$, 其在 研发创新过程中发挥着双重角色, 与 $s$ 个中间投入 $I_{t j}$ $(t=1,2, \cdots \cdots, s)$ 共同作为第二阶段的投入, 最终产生 $h$ 个 最终产出 $Y_{r j}(r=1,2, \cdots \cdots, h)$ 。此外, 基于研发创新过程中 的阶段滞后特性，分别对第一阶段投入指标、第一阶段产 出指标（或第二阶段投入指标）、第二阶段产出指标采用 第 $T$ 年，第 $T+1$ 年，第 $T+2$ 年 $[5]$ 。

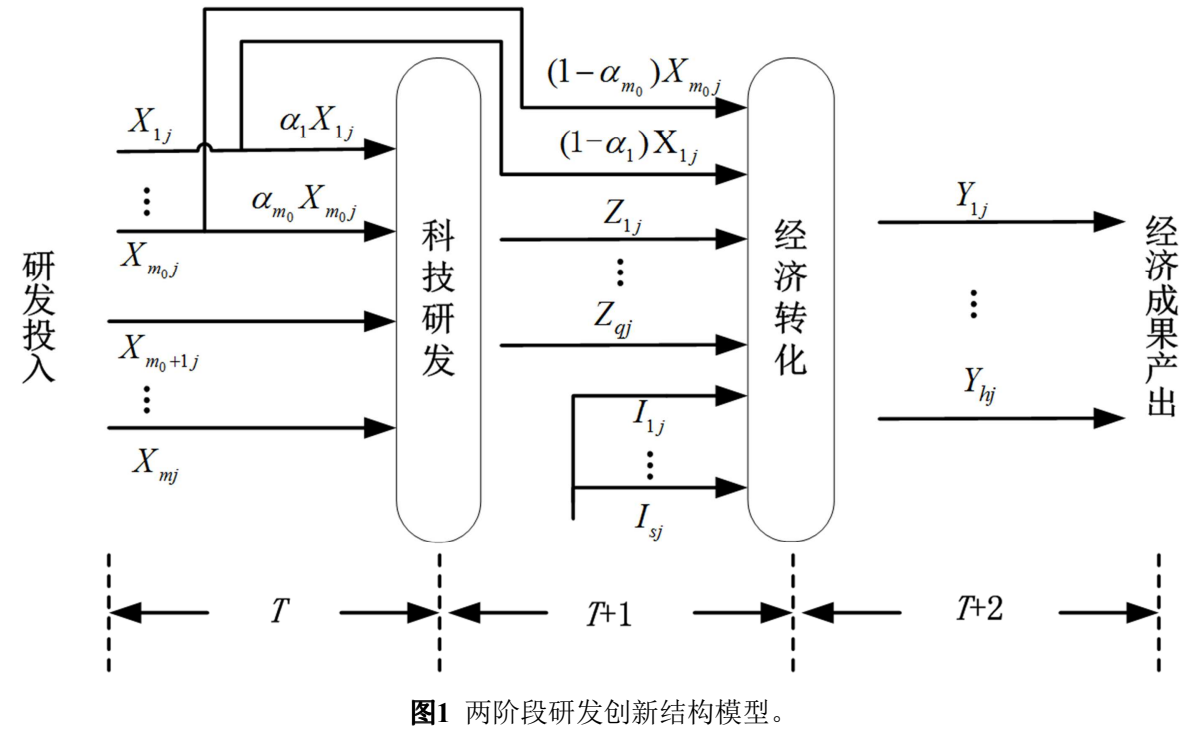




\section{3. 改进的两阶段DEA模型构建}

改进两阶段DEA模型相较现有两阶段DEA模型主要 有以下两个改进之处。一方面, 针对现有两阶段DEA模型 [13]中只能将DMU简单分为处于效率前沿面的DMU（效 率评价得分为 1）与无效DMU（效率评价得分小于1）两 类, 无法对DMU单元排序的问题, 改进的两阶段DEA模 型借鉴Anderson和Petersen[12]提出的将有效DMU从效率 前沿面分离出去的思想构建超效率DEA模型。另一方面, 改进两阶段DEA模型基于可变报酬（VRS）假设，借鉴 Banker提出的BCC模型[14]与陈凯华等[3]的处理方法, 在 模型中引入分离变量 $\theta$ 来反映规模效应的影响, 以消除不 变规模报酬（CRS）假设的效率评价误差。

$$
\begin{array}{r}
\text { 不妨 命 } e_{k}=\left(\sum_{p=1}^{q} \varphi_{p}^{1} Z_{p k}+\sum_{r=1}^{h} \mu_{r} Y_{r k}-\theta_{k}\right) /\left(\sum_{i=1}^{m_{0}} \lambda_{i}^{1} \alpha_{i} X_{i k}+\right. \\
\left.\sum_{i=1}^{m_{0}} \lambda_{i}^{2}\left(1-\alpha_{i}\right) X_{i k}+\sum_{l=m_{0}+1}^{m} \rho_{l} X_{l k}+\sum_{p=1}^{q} \varphi_{p}^{2} Z_{p k}+\sum_{t=1}^{s} \omega_{t} I_{t k}\right) \text { 。依据最 }
\end{array}
$$

优化思想构建DEA比率模型的思想, 被评估单元DMU $\mathrm{U}_{j}$ 的 整体研发效率与整体约束条件可以表示为如下 (1)、（2） 式所示（投入导向DEA模型）。

$$
\max e_{k}
$$

$$
\text { s.t. }\left\{\begin{array}{l}
e_{k} \leq 1, j=1,2 \cdots \cdots N, j \neq k \\
0<\alpha_{i} \leq 1 \\
\lambda_{i}^{1}, \lambda_{i}^{2}, \rho_{l}, \varphi_{p}^{1}, \varphi_{p}^{2}, \omega_{t}, \mu_{r} \geq 0 \\
i=1,2, \cdots \cdots, m_{0} \\
1=m_{0}+1, \cdots \cdots, m \\
p=1,2, \cdots \cdots, q \\
\mathrm{t}=1,2, \cdots \cdots, \mathrm{s} \\
\mathrm{r}=1,2, \cdots \cdots, h
\end{array}\right.
$$

（1）式为目标函数, $e_{k}$ 表示被决策单元 $\mathrm{DMU}_{k}$ 的总体 效率值, 规划目标为实现可行域内 $e_{k}$ 的最大化, 同时获得 决策变量 $\left(\alpha_{i}, \lambda_{i}{ }^{1}, \lambda_{i}{ }^{2}, \rho_{l}, \varphi_{p}{ }^{1}, \varphi_{p}{ }^{2}, \omega_{t}, \mu_{r}, \theta_{k}\right)$ 的最优 取值组合。其中, 式（1）中各决策变量分别表示对应不 同投入 (或产出) 指标的权重结构。特别地, $\theta_{k}$ 表示VRS 假设下反映规模效应的分离变量, 模型为面向投入导向, 故在产出中予以剔除（或增加）[14]。考虑中间产出 $Z_{p j}$ 的 双重角色, 分别用 $\varphi_{p}{ }^{1}$ 和 $\varphi_{p}{ }^{2}$ 两个决策变量度量 $Z_{p k}$ 在两个阶 段的不同权重结构。同时由于 $X_{i j}$ 在两个阶段的作用不同， 采用 $\lambda_{i}{ }^{1}$ 和 $\lambda_{i}{ }^{2}$ 两个决策变量分别表示两部分投入在各自阶 段的权重。

(2) 式所反映的约束条件是为实现 $D M U_{k}$ 总体效率取 值最优化需满足的整体约束。第一个不等式约束使得在对 $\mathrm{DMU}_{k}$ 进行评价时, 取消了传统DEA评价中 $e_{k} \leq 1$ 的约束, 而保留了对其他非决策单元效率取值 $\left(e_{j}, j \neq k\right)$ 介于 0 与 1 之间的约束, 研究 $[13,15]$ 表明这种超效率DEA评价方法在 基本不影响效率有效性评价的基础上, 实现了对处于效率 有效前沿面上的决策单元的排序, 使得结果更具有参考意
义。鉴于研发创新过程被分为两个阶段, 在进行效率评价 时还应当考虑两个子过程的局部最优化条件。于是, 将 (1), (2) 所示数学规划改进为基于改进两阶段DEA的研发创 新效率评价模型, 如下（3）、（4）所示。

$$
\max e_{k}
$$

$$
\text { s.t. }\left\{\begin{array}{l}
\zeta_{1} \leq 1, j=1,2 \cdots \cdots, N ; j \neq k \\
\zeta_{2} \leq 1, j=1,2 \cdots \cdots, N ; j \neq k \leq 1 \\
0<\alpha_{i} \leq 1 \\
\lambda_{i}^{1}, \lambda_{i}^{2}, \rho_{l}, \varphi_{p}^{1}, \varphi_{p}^{2}, \omega_{t}, \mu_{r} \geq 0 \\
i=1,2, \cdots \cdots, m_{0} \\
1=m_{0}+1, \cdots \cdots, m \\
p=1,2, \cdots \cdots, q \\
t=1,2, \cdots \cdots, s \\
r=1,2, \cdots \cdots, h
\end{array}\right.
$$

$$
\begin{array}{r}
\text { 其中, } \zeta_{1}=\left(\sum_{p=1}^{q} \varphi_{p}^{1} Z_{p j}-\theta_{k}^{1}\right) /\left(\sum_{i=1}^{m_{0}} \lambda_{i}^{1} \alpha_{i} X_{i j}+\sum_{l=m_{0}+1}^{m} \rho_{l} X_{l j}\right), \\
\zeta_{2}=\left(\sum_{r=1}^{h} \mu_{r} Y_{r j}-\theta_{k}^{2}\right) /\left(\sum_{i=1}^{m_{0}} \lambda_{i}^{2}\left(1-\alpha_{i}\right) X_{i j}+\sum_{p=1}^{q} \varphi_{p}^{2} Z_{p j}+\sum_{t=1}^{s} \omega_{t} I_{t j}\right) 。
\end{array}
$$

$\theta_{k}{ }^{1}$ 与 $\theta_{k}{ }^{2}$ 分别代表两个子过程中反映规模效应的分离变量, $\theta_{k}=\theta_{k}{ }^{1}+\theta_{k}{ }^{2}$; （2）式中约束可由两子阶段约束线性表示,

在 (4) 式约束条件族中省去。

结合上述分析, 改进后的两阶段DEA效率评价模型在 充分考虑中间产出双重作用与子过程效率局部最优化条 件的基础上, 实现了对有效DMU的排序。从求解角度, 该模型为分式规划, 考虑将上述分式规划转化为线性规划。 命 $\quad \xi=1 /\left(\sum_{i=1}^{m_{0}} \lambda_{i}^{1} \alpha_{i} X_{i k}+\sum_{i=1}^{m_{0}} \lambda_{i}^{2}\left(1-\alpha_{i}\right) X_{i k}+\sum_{l=m_{0}+1}^{m} \rho_{l} X_{l k}+\right.$ $\left.\sum_{p=1}^{q} \varphi_{p}^{2} Z_{p k}+\sum_{t=1}^{s} \omega_{t} I_{t k}\right), \zeta_{3}=\sum_{i=1}^{m_{0}} \Omega_{i}^{1} X_{i k}+\sum_{i=1}^{m_{0}} \Lambda_{i}^{2} X_{i k}-\sum_{i=1}^{m_{0}} \Omega_{i}^{2} X_{i k}+$ $\sum_{l=m_{0}+1}^{m} B_{l} X_{l k}+\sum_{p=1}^{q} \psi_{p}^{2} Z_{p k}+\sum_{t=1}^{s} W_{t} I_{t k}, \zeta_{4}=\sum_{i=1}^{m_{0}} \Lambda_{i}^{2} X_{i j}-\sum_{i=1}^{m_{0}} \Omega_{i}^{2} X_{i j}+$ $\sum_{p=1}^{q} \psi_{p}^{2} Z_{p j}+\sum_{t=1}^{s} W_{t} I_{t j}-\sum_{r=1}^{h} U_{r} Y_{r j}+\Theta_{k}^{2} \quad, \quad \zeta_{5}=\sum_{i=1}^{m_{0}} \Omega_{i}^{1} X_{i j}+$ $\sum_{l=m_{0}+1}^{m} B_{l} X_{l j}-\sum_{p=1}^{q} \psi_{p}^{1} Z_{p j}+\Theta_{k}^{1}$ 。利用Charnes-Cooper转换, 分式规划可化简为等价的数学规划 (5) 所示。

$$
\max e_{k}=\sum_{p=1}^{q} \psi_{p}^{1} Z_{p k}+\sum_{r=1}^{h} U_{r} Y_{r k}-\Theta_{k}^{1}-\Theta_{k}^{2}
$$




$$
\text { s.t. }\left\{\begin{array}{l}
\zeta_{3}=1 \\
\zeta_{4} \geq 0, j=1,2, \cdots \cdots, N n j \neq k \\
\zeta_{5} \geq 0, j=1,2, \cdots \cdots, N ; j \neq k \\
\varepsilon \leq \Omega_{i}^{2} \leq \Lambda_{i}^{2} \\
\Omega_{i}^{1}, B_{l}, \psi_{p}^{1}, \psi_{p}^{2}, W_{t}, U_{r} \geq \varepsilon \\
i=1,2, \cdots \cdots, m_{0} \\
1=m_{0}+1, \cdots \cdots, m \\
p=1,2, \cdots \cdots, q \\
t=1,2, \cdots \cdots, s \\
r=1,2, \cdots \cdots, h
\end{array}\right.
$$

其中, $\Lambda_{i}^{1}=\xi \lambda_{i}{ }^{1}, \Lambda_{i}{ }^{2}=\xi \lambda_{i}{ }^{2}, B_{l}=\xi \rho_{l}, \Psi_{p}^{1}=\xi \varphi_{i}{ }^{1}, \Psi_{p}^{2}=\xi \varphi_{i}^{2}$, $W_{t}{ }^{1}=\xi \omega_{i}{ }^{1}, \quad U_{r}{ }^{1}=\xi \mu_{r}{ }^{1}, \quad \Theta_{\mathrm{k}}{ }^{1}=\xi \theta_{i}{ }^{1}, \quad \Theta_{\mathrm{k}}{ }^{2}=\xi \theta_{i}{ }^{2}, \quad \Omega{ }_{i}{ }^{1}=\alpha_{i} \Lambda_{i}{ }^{1}, \Omega$ ${ }_{i}^{2}=\alpha_{i} \Lambda_{i}^{2}$ 。 $\varepsilon$ 为非阿基米德无穷小，(5) 中所示规划存在可 行解时, $\varepsilon$ 取值受投入产出规模的限定 [3]。

\section{4. 实证研究}

\section{1. 改进两阶段DEA研发创新效率评价模型的指标设计}

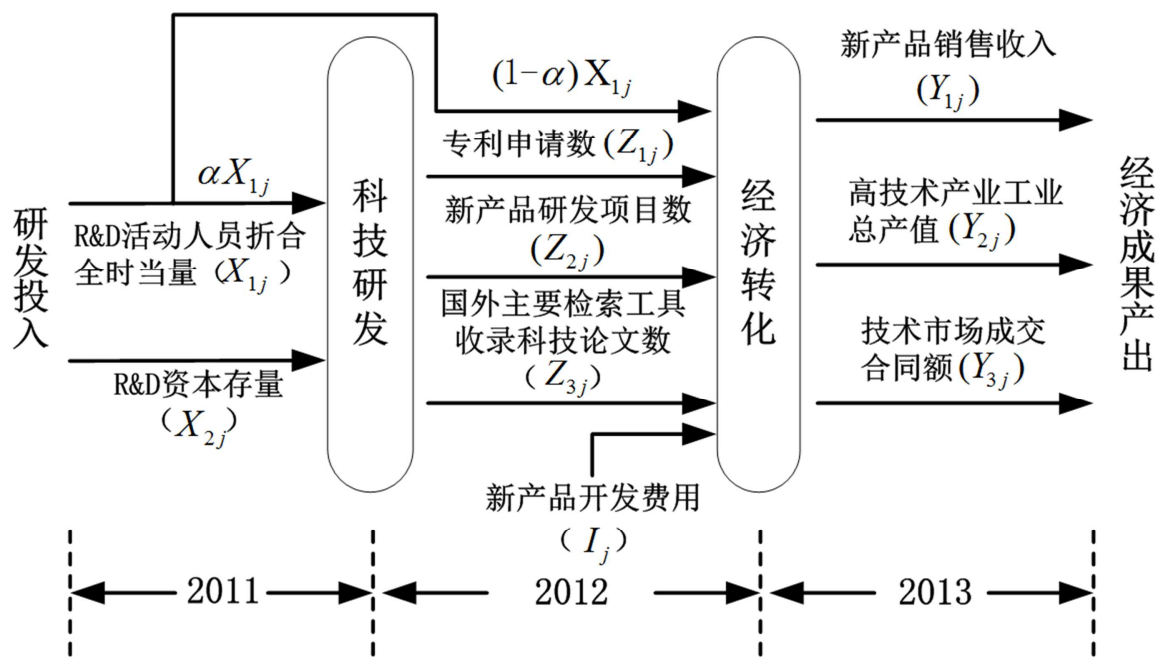

图2 改进两阶段DEA模型的指标设计。

该部分将根据上述构建的模型对中国大陆30省区（西 藏由于数据缺乏，分析时将其略去）研发创新效率进行研 究，并对中国华北、东北、华东、中南、西南、西北六大 区域进行创新研发效率的比较分析, 以对实践研究提供一 定的参考价值。文中数据主要来源于2010 2014年《中国 科技统计年鉴》和《中国高科技产业统计年鉴》。研发创 新过程分为科技研发和经济转化两个子过程, 各阶段投入 和产出指标的选取主要参考李婧[16]、冯峰[4]、马志军[5] 等学者的研究, 具体指标设计如下图2所示。

特别地, 由于 $R \& D$ 经费内部支出是一项流量指标, 仅 能反映当期创新投入水平, 不能反映创新活动对后期知识 生产影响的延续性[14], 因此, 本文选用R\&D资本存量取 代R\&D经费内部支出作为一项研发投入指标, 以更符合实 际要求。本文借鉴石崩然等的研究 [18], 采用永续盘存法
由线性规划 (5) 和 (6) 可解得决策变量 $\left(\Omega_{i}{ }^{1}, \Omega_{i}{ }^{2}\right.$, $\left.\Lambda_{i}^{2}, B_{l}, \Psi_{p}{ }^{1}, \Psi_{p}{ }^{2}, W_{t}, U_{r}, \Theta_{\mathrm{k}}{ }^{1}, \Theta_{\mathrm{k}}{ }^{2}\right)$ 的最优解组合 (7) 式和（8）式, 并可进一步代入（7）式和（8）式解得子 过程效率值。

$$
\begin{gathered}
e_{k}^{1}=\frac{\sum_{p=1}^{q} \psi_{p}^{1} Z_{p k}-\Theta_{k}^{1}}{\sum_{i=1}^{m_{0}} \Omega_{i}^{1} X_{i k}+\sum_{l=m_{0}+1}^{m} B_{l} X_{l k}} \\
e_{k}^{2}=\frac{\sum_{r=1}^{h} U_{r} Y_{r k}-\Theta_{k}^{2}}{\sum_{i=1}^{m_{0}} \Lambda_{i}^{2} X_{i k}-\sum_{i=1}^{m_{0}} \Omega_{i}^{2} X_{i k}+\sum_{p=1}^{q} \psi_{p}^{2} Z_{p k}+\sum_{t=1}^{s} W_{t} I_{t k}}
\end{gathered}
$$

来核算 $R \& D$ 资本存量, 按照国际上平均滞后假定, 选取滞 后期为 1 , 贴现系数为 1 , 其计算公式为:

$$
\left\{\begin{array}{l}
X_{2 j}{ }^{(\mathrm{t})}=(1-\delta) X_{2 j}{ }^{(\mathrm{t}-1)}+O_{j}^{(t)} \\
X_{2 j}{ }^{(0)}=\frac{O_{j}^{(0)}}{g+\delta}
\end{array}\right.
$$

式 (9) 中, $X_{2 j}{ }^{(t)}, X_{2 j}{ }^{(t)}, X_{2 j}{ }^{(0)}$ 分别代表第 $j$ 个省区第 $t$ 、 $t-1$ 期和基期的资本存量, $\delta$ 为折旧率，借鉴吴延兵 [14]、 李婧 [13 等人的做法, 取 $\delta=15 \%$ 。 $O_{j}^{(t)}, O_{j}^{(t)}$ 分别代表第 $j$ 个 省区第 $t$ 期和基期的实际 $R \& D$ 支出, 本文主要考察不同省 份间的研发效率差异，且为分析期选取较短（5年），不 失一般性, 将该项指标选取为 $R \& D$ 名义支出, 而忽略 $R \& D$ 价格支出指数的影响。 $g$ 为考察期内 $R \& D$ 经费支出的平均 增长率, 按照可比价格计算。进行数据处理时, 选取2009 
年为基期, 考察2009 2013年研发支出的数据变化情况以 获得2011年度各省区的R\&D资本存量指标数据。

\section{2. 研发创新效率的省际差异性分析}

运用MATLAB7.8对 30 个省份的数据进行处理, 可以 获得不同省份改进两阶段DEA模型下的综合研发创新效 率评价排名, 进一步对改进两阶段DEA区域研发创新效率 评价模型的结果进行分析与计算, 可以获得相应两阶段综 合研发创新效率值与子过程效率值如下表1所示。

表1 基于改进两阶段DEA模型的分省份综合效率与子过程效率比较分析。

\begin{tabular}{|c|c|c|c|c|c|c|c|}
\hline 排名 & 省市 & e & $\mathrm{e}^{1}$ & $\mathrm{e}^{2}$ & $\Theta_{\mathrm{k}}{ }^{1}$ & $\Theta_{k}{ }^{2}$ & $\alpha$ \\
\hline 1 & 江苏 & 3.953 & 4.764 & 2.198 & 92.500 & 97.827 & 1.000 \\
\hline 2 & 广东 & 3.287 & 1.520 & 4.339 & 47.380 & 58.010 & 1.000 \\
\hline 3 & 北京 & 2.644 & 2.698 & 1.046 & 444.739 & 36.096 & 1.000 \\
\hline 4 & 上海 & 1.686 & 0.997 & 1.881 & 57.326 & 24.220 & 1.000 \\
\hline 5 & 海南 & 1.642 & 0.837 & 1.860 & -1.085 & 0.000 & 0.998 \\
\hline 6 & 甘肃 & 1.624 & 1.103 & 1.636 & -1.278 & 0.000 & 1.000 \\
\hline 7 & 福建 & 1.623 & 1.918 & 0.883 & 0.498 & 0.000 & 1.000 \\
\hline 8 & 浙江 & 1.532 & 1.626 & 0.976 & -0.007 & 0.000 & 1.000 \\
\hline 9 & 重庆 & 1.251 & 1.100 & 1.417 & 0.000 & 0.034 & 0.000 \\
\hline 10 & 江西 & 1.218 & 1.230 & 1.130 & 0.777 & -54.135 & 1.000 \\
\hline 11 & 广西 & 1.202 & 1.382 & 0.931 & -32.455 & -84.290 & 1.000 \\
\hline 12 & 四川 & 1.145 & 1.158 & 0.985 & 0.300 & 0.000 & 1.000 \\
\hline 13 & 吉林 & 1.110 & 1.120 & 1.000 & 0.149 & 0.000 & 1.000 \\
\hline 14 & 黑龙江 & 1.071 & 1.101 & 1.000 & 0.275 & 0.000 & 1.000 \\
\hline 15 & 陕西 & 1.053 & 1.076 & 0.994 & 0.013 & 0.000 & 1.000 \\
\hline 16 & 河南 & 1.021 & 0.780 & 1.903 & 0.000 & 5.118 & 1.000 \\
\hline 17 & 内蒙古 & 1.019 & 0.599 & 2.177 & 0.000 & 1.424 & 1.000 \\
\hline 18 & 天津 & 1.000 & 1.009 & 1.000 & 133.697 & 130.033 & 1.000 \\
\hline
\end{tabular}

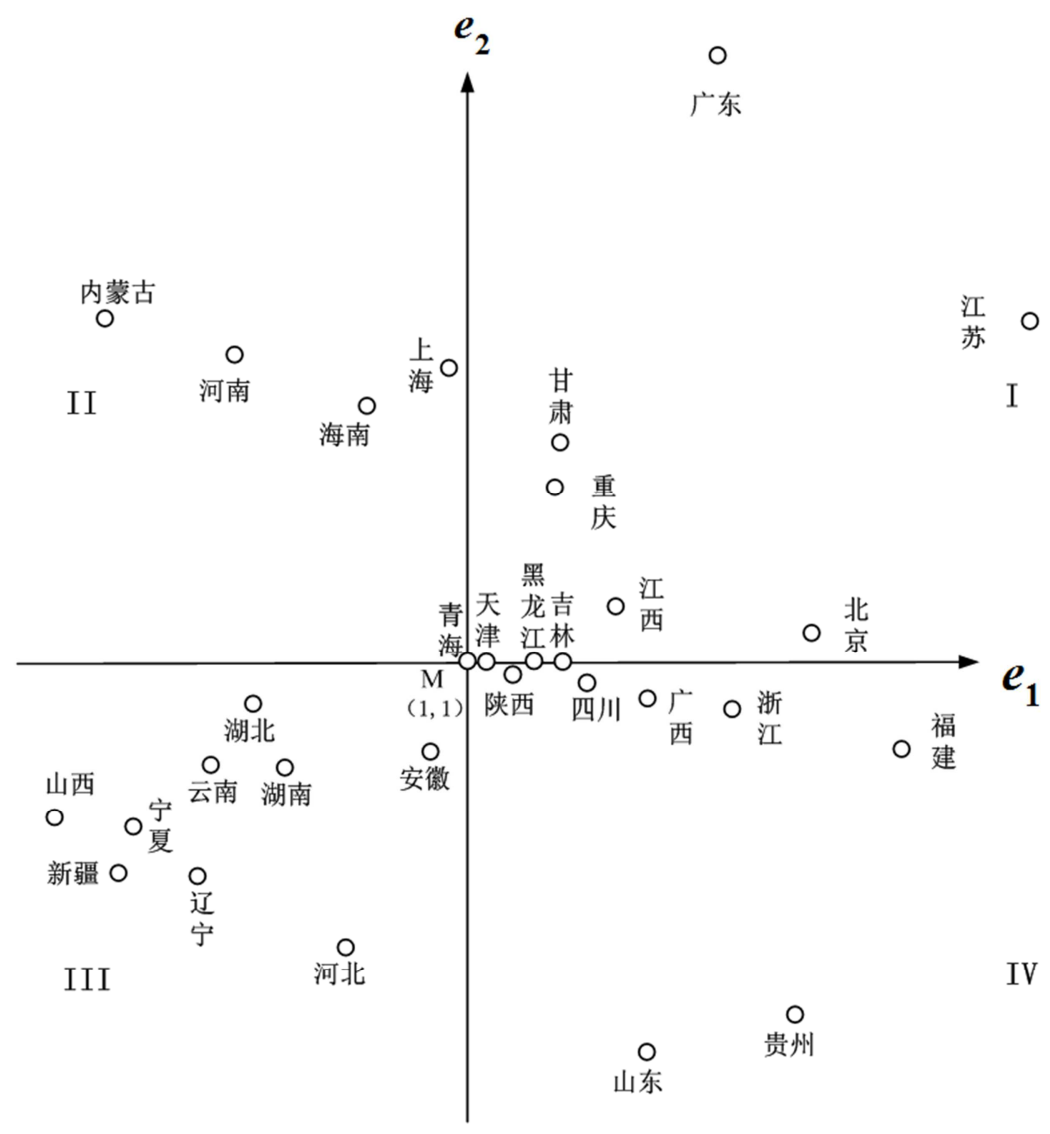

图3 各省份两阶段研发创新效率分布图。

\begin{tabular}{llllllll}
\hline 排名 & 省市 & $\mathbf{e}$ & $\mathbf{e}^{\mathbf{1}}$ & $\mathbf{e}^{\mathbf{2}}$ & $\boldsymbol{\Theta}_{\mathbf{k}}{ }^{1}$ & $\boldsymbol{\Theta}_{\mathbf{k}}{ }^{2}$ & $\boldsymbol{\alpha}$ \\
\hline 19 & 青海 & 1.000 & 1.000 & 1.000 & 0.000 & -53.499 & 1.000 \\
20 & 安徽 & 0.950 & 0.991 & 0.880 & 0.046 & 0.000 & 1.000 \\
21 & 贵州 & 0.940 & 1.921 & 0.461 & 0.205 & 0.000 & 0.999 \\
22 & 湖北 & 0.850 & 0.747 & 0.931 & 0.168 & 0.000 & 0.979 \\
23 & 湖南 & 0.810 & 0.809 & 0.861 & 0.118 & 0.000 & 1.000 \\
24 & 云南 & 0.790 & 0.742 & 0.905 & -0.030 & 0.000 & 1.000 \\
25 & 山东 & 0.780 & 1.312 & 0.453 & 0.000 & 0.423 & 1.000 \\
26 & 辽宁 & 0.770 & 0.767 & 0.780 & -0.040 & 0.000 & 1.000 \\
27 & 宁夏 & 0.750 & 0.635 & 0.845 & 0.000 & -0.672 & 0.000 \\
28 & 新疆 & 0.720 & 0.632 & 0.801 & -0.051 & 0.000 & 0.994 \\
29 & 河北 & 0.708 & 0.855 & 0.610 & 0.046 & 0.000 & 1.000 \\
30 & 山西 & 0.681 & 0.595 & 0.858 & 0.000 & 0.104 & 0.000 \\
均值 & & 1.203 & 1.234 & 1.225 & - & - & - \\
\hline
\end{tabular}

注: e, $\mathrm{e}^{1}, \mathrm{e}^{2}$ 分别代表综合研发创新效率和两个子过程研发创新效率; $\Theta_{\mathrm{k}}{ }^{\mathrm{i}}$ 与规模效应影响参数 $\theta_{\mathrm{k}}{ }^{\mathrm{i}}$ 正相关, 用来表征规模效应对效率评价的影响, $\mathrm{i}=1,2$ 。

由表1可以看出, 改进的两阶段DEA模型实现了在不 改变无效率决策单元 (省市) 的效率排名的前提下, 填补 了传统两阶段DEA模型无法对有效决策单元进行效率排 序与深入分析的空白。下面主要从三个角度分析表1所得 的效率评价结果与参数结果。首先, 从全国的两阶段综合 科技研发创新效率角度, 有 19 个省份呈现效率有效性 $\left(e_{k} \geq 1\right)$, 总体综合效率均值为 $1.203>1$, 说明考察期 （2011 2013年）内总体科技研发创新效率总体表现良好, 其中 1.203 的含义为当初始投入增加 $20.3 \%$ 时, 总体综合效 率仍保持有效水平 $(\geq 1)$ 。 
其次, 从各省份的综合效率评价结果和分阶段子过程 的研发创新效率结果角度，省份间呈现不同特征。为了更 加清晰地分析不同省份的研发创新效率特性, 图3分别以 $\mathrm{e}_{1}$ 和 $\mathrm{e}_{2}$ 为横、纵坐标轴, 以 $M(1,1)$ 为轴心点, 划分为 四个象限对 30 个省份的研发创新效率进行描述。象限 $\mathrm{I}$ 代 表两个子阶段均效率均大于 1 的“优性”象限, 象限III代表 两子阶段效率水平的“劣性”象限, 位于象限II和IV的省份 则呈现两子阶段效率中其中仅某一子过程效率大于 1 的 “次优性”特征。从图1各省份点的分布来看, 呈现“优性” 特征的省份占到 $20 \%$ 30\%左右，比例并不是很高，但“次 优性” (II、IV) 象限的点向其转化的难度较小、可实现性 较强, 因此可以借助区域间研发合作与集群创新等区域联 动力, 以及区域产业链条建设、孵化平台建设等举措推动 这些省份向“优性”象限转化。针对位于“劣性”（III）象限 的几个省份, 短期内实现其向“优性”象限转化的实施难度 很大, 因此可以考虑结合其自身区位特点、产业优势等, 优先选择进行科技研发效率的提升或经济转化效率的提 升, 依靠“劣性”到“次优”，再到“优性”的分步发展路径， 最终实现总体的高效、平衡发展。在实施策略上, 由于某 些地区，如宁夏、新疆等几个西部省份，区域内“优性”省 份较少, 难以单靠区域联动与合作实现对“劣性”省份的带 动, 因此需要借助广东、江苏、北京等研发创新效率领先 地区的帮扶与指导, 加上政府的政策与经济支持, 共同推 动这些省份的研发创新效率提升。

最后, 从规模效应对各省份的研发创新效率评价结果 的影响角度分析, 可以看出规模效应的影响存在较明显的 差异性。根据模型的构建过程, 参数分别与测度两阶段规 模效应的指标 $\theta_{\mathrm{k}}{ }^{1} 、 \theta_{\mathrm{k}}{ }^{2}$ 成正比例关系, 不失一般性, 规模 效应的影响可以借助 $\Theta_{\mathrm{k}}{ }^{1} 、 \Theta_{\mathrm{k}}{ }^{2}$ 来表征。 $\Theta_{\mathrm{k}}{ }^{\mathrm{i}}(\mathrm{i}=1,2)$ 为正, 代表规模效应为负, 为剔除项; 反之, 若其值为负, 则代 表规模效应为正, 为增加项。从表 1 的结果来看, 两个子 过程的规模效应对各省研发创新效率的评价有一定影响, 尤其是广东、天津、江苏等省份, 将这一指标的引入, 使 得各省份的研发创新效率的评价结果更为客观。

\section{3. 研发创新效率的区域差异性分析}

为了更加清晰地比较研发创新效率的区域差异, 为区 域定位与发展战略制定提供参考, 下对中国六个行政区域, 即: 华北 (京、津、冀、晋、蒙)、东北（黑、吉、辽）、 华东（沪、苏、浙、皖、闽、贑、鲁）、中南（豫、鄂、 湘、粤、桂、琼）、西南（渝、川、贵、云）和西北（陕、 甘、青、宁、疆）六个区域进行研发创新效率的区域比较, 结果如下表2所示。

表2 区域研发创新效率比较。

\begin{tabular}{llllllll}
\hline \multirow{2}{*}{ 编号 } & \multirow{2}{*}{ 区域 } & \multicolumn{2}{l}{ 综合效率 } & \multicolumn{2}{l}{ 第一阶段效率 } & \multicolumn{2}{l}{ 第二阶段效率 } \\
\cline { 3 - 8 } & $\mathbf{e}$ & 标准差 & $\mathbf{e}^{\mathbf{1}}$ & 标准差 & $\mathbf{e}^{\mathbf{2}}$ & 标准差 \\
\hline 1 & 华北 & 1.212 & 0.815 & 1.151 & 0.882 & 1.138 & 0.605 \\
2 & 东北 & 0.984 & 0.186 & 0.996 & 0.199 & 0.927 & 0.127 \\
3 & 华东 & 1.677 & 1.061 & 1.834 & 1.334 & 1.200 & 0.616 \\
4 & 中南 & 1.469 & 0.941 & 1.013 & 0.344 & 1.804 & 1.331 \\
5 & 西南 & 1.031 & 0.206 & 1.230 & 0.496 & 0.942 & 0.392 \\
6 & 西北 & 1.029 & 0.364 & 0.889 & 0.236 & 1.055 & 0.336 \\
\hline
\end{tabular}

表 2 的结果可以从区域效率均值与标准差两个方面分 析。一方面, 从六个区域的效率均值看, 华东地区研发创 新效率水平最高, 中南、华北的综合效率和分阶段效率均 值水平也都大于 1 , 呈现“优性特征”, 西南、西北地区综 合效率水平大于 1 , 但呈现科技研发效率或经济转化效率 小于 1 的“次优性”表现，东北地区研发创新效率的均值水 平最低。

另一方面, 标准差指标测度了同一区域内不同省份偏 离均值水平的波动程度, 也即同一区域省份间研发创新效 率的差异性程度。从表 2 的分析结果来看, 东北地区各省 份差异性最小, 研发创新效率较为近似; 西南、西北地区 标准差也较小, 区域内省间差异较小; 而华东、中南、华 北地区虽然研发创新效率总体表现优异, 但区域内省间效 率差异性较大, 呈现不均衡的特征。

\section{5. 结论}

本文将科技研发创新效率分为科技研发阶段和经济 转化阶段，考虑了中间产出的“双重角色”，并通过赋予 “两个角色”不同权重来测度中间产出在两阶段的不同作 用。此外, 基于传统两阶段效率评价模型进行了如下两 个方面的改进：（1）引入超效率 $\mathrm{BCC}$ 模型, 修正了传统 模型不能对有效决策单元排序的问题; (2) 引入测度规 模效应的参数, 剔除了规模效应对研发创新效率评价结 果的影响。

为验证改进的两阶段DEA研发创新效率评价模型的 可行性与适用性, 本文对 30 个省份进行了两阶段研发创新 效率评价的实证分析, 通过与传统两阶段DEA评价模型的 评价结果比较, 发现改进的两阶段DEA研发创新效率的评 价结果在未改变非有效决策单元 (省份) 排序的前提下, 实现了有效决策单元 (省份) 的排序, 评价结果较具参考 价值。

通过区域间效率评价均值比较与标准差比较, 对区域 间水平总体差异与区域内省间差异进行了分析, 研究发现: (1) 华东地区研发创新效率水平最高, 中南、华北的综 合效率和分阶段效率均高, 西南、西北地区综合效率水平 高, 但科技研发效率或经济转化效率低, 东北地区研发创 新效率最低。（2）东北地区各省份差异性最小, 研发创 新效率较为近似; 西南、西北地区标准差也较小, 区域内 省间差异较小; 而华东、中南、华北地区虽然研发创新效 率总体表现优异, 但区域内省间效率差异性较大, 呈现不 均衡的特征。本文的研究结论可为区域发展提供一定的参 考。

\section{致谢}

本文为国家社科基金项目《基于消费者选择行为的 新能源汽车研发财政资助政策研究》(17XGL008)和重庆 市社会科学规划重点项目《重庆市融入国家“一带一路” 战略的思路及对策研究》(2015ZDJJ04)的阶段性成果之 


\section{参考文献}

[1] Yao Chen, Liang Liang, Feng Yang et al. Evaluation of information technology investment: a data envelopment analysis approach[J]. Computers Operations Research, 2006, 33(3):1368-1379.

[2] Kao C, Hwang S N. Efficiency measurement for network systems: IT impact on firm performance [J]. Decision Support Systems, 2010, 48(3):437-446.

[3] 陈凯华,官建成.共享投入型关联两阶段生产系统的网络 DEA 效率测度与分解 [J]. 系统工程理论与实 践,2011,31(7):1211-1221。

[4] 冯锋,张雷勇,高牟等.两阶段链视角下科技投入产出链效率 研究——来自我国29个省市数据的实证 $[\mathrm{J}]$.科学学与科学 技术管理,2011,32(8):33-38。

[5] 马建峰,何枫.包含共享投入与自由中间产出的技术创新两 阶段DEA效率评价[J].系统工程,2014,32(1):1-9。

[6] 刘瑞翔,吕大雪,骆依.不良贷款约束下我国商业银行效率的 测评--基于两阶段DEA模型的分析 [J].南京审计大学学报, 2016,13(6):41-50。

[7] 黄薇.中国保险机构资金运用效率研究:基于资源型两阶段 DEA模型[J].经济研究,2009,(8):37-49。

[8] 王美强,李勇军.具有双重角色和非期望要素的供应商评价 两阶段DEA模型[J].中国管理科学,2016,24(12):91-97。

[9] 代明,刘可新,陈俊.中国高技术产业研发创新效率研究 [J].中 国科技论坛,2016,(1):5-10。

[10] Yang G L, Fukuyama H, Song $Y$ Y. Measuring the inefficiency of Chinese research universities based on a two-stage network DEA mode 1[J]. Journal of Informetrics, 2018, 12(1):10-30.

[11] Izadikhah M, Tavana M, Caprio D D, et al. A Novel Two-Stage DEA Production Model with Freely Distributed Initial Inputs and Shared Intermediate Outputs [J]. Expert Systems with Applications, 2018, In Press:213-230.

[12] Andersen P., Petersen N. C. A Procedure for Ranking Efficient Units in Data Envelopment Analysis [J]. Management Science, 1993, (39):1261-1264.

[13] 吉生保,席艳玲,赵祥.中国农业上市公司绩效评价一一基于 SORM-BCC 超效率模型和Malmquist的DEA-Tobit分析 [J]. 农业技术经济,2012(3):114-127。

[14] R. D. Banker, A. Charnes, W. W. Cooper. Some Models for Estimating Technical and Scale Inefficiencies in Data Envelopment Analysis [J]. Management Science, 1984, 30(9):1078-1092.

[15] 王金祥.基于超效率DEA模型的交叉效率评价方法 [J].系统 工程,2009,27(6):115-118。

[16] 李婧.基于动态空间面板模型的中国区域创新集聚研究 [J]. 中国经济问题,2013,(6):56-66。

[17] 吴延兵.中国地区知识生产效率测算 [J]. 财经研 究,2008,(10):4-14。

[18] 石崩然,赵顺龙.R\&D资本存量与我国高技术产业若干指标 的相关性分析 [J].科学学与科学技术管理,2010,(1):107-111。 